\title{
Stabilization of Discrete-Time Planar Switched Linear Systems with Impulse
}

\author{
Yanli Zhu ${ }^{1,2}$ and Yuangong Sun ${ }^{1}$ \\ ${ }^{1}$ School of Mathematical Sciences, University of Jinan, Jinan, Shandong 250022, China \\ ${ }^{2}$ School of Automation and Electrical Engineering, University of Jinan, Jinan, Shandong 250022, China \\ Correspondence should be addressed to Yuangong Sun; sunyuangong@yahoo.cn
}

Received 20 January 2013; Accepted 3 April 2013

Academic Editor: Hua Su

Copyright (C) 2013 Y. Zhu and Y. Sun. This is an open access article distributed under the Creative Commons Attribution License, which permits unrestricted use, distribution, and reproduction in any medium, provided the original work is properly cited.

\begin{abstract}
We study the stabilization problem of discrete-time planar switched linear systems with impulse. When all subsystems are controllable, based on an explicit estimation on the state transition matrix, we establish a sufficient condition such that the switched impulsive system is stabilizable under arbitrary switching signal with given switching frequency. When there exists at least one uncontrollable subsystem, a sufficient condition is also given to guarantee the stabilization of the switched impulsive system under appropriate switching signal.
\end{abstract}

\section{Introduction}

Recent years have witnessed a rapid progress for switched systems, for example, see monographs [1-3] and survey papers $[4,5]$. As usual, a switched system means a type of hybrid dynamic system that consists of a family of continuous-time (discrete-time) subsystems and a switching signal, which determines the switching between subsystems. It is well known that switched systems have a deep background in engineering such as computer disk system [6], robotics [7], power systems [8], air traffic management [9], and automated vehicles [10].

During the last three decades, there is an increasing interest on the stability analysis for switched systems. For stability issues; one important problem is to find conditions that guarantee asymptotic stability of the switched system for arbitrary switching signal. Such a problem is usually studied by using a common Lyapunov functional approach, especially by using a common quadratic Lyapunov functional approach [11-14]. A multiple Lyapunov functional method was used to study the stability of switched systems with delays in [15].

For systems that switch among a finite set of controllable linear systems, the stabilization problem of continuous-time switched systems with arbitrary switching frequency was studied in $[16,17]$ by developing an improved estimation on transition matrices. Very recently, the results in $[16,17]$ were further extended to switched systems with impulses and perturbations [18]. So far, the stability and stabilization problems for switched systems were studied in [19-31], to name a few.

In this paper, motivated by the work in [16-18], we study the stabilization problem of discrete-time planar switched linear systems under impulse and arbitrary switching signal with given switching frequency. When all subsystems are controllable, we obtain a discrete analogue of the main result in [17]. We also consider the case when there exist both controllable subsystems and uncontrollable subsystems. Before giving our main results, we first establish an estimation on the transition matrix for each controllable subsystem, which plays a key role in the stabilization problem of the switched system. For the uncontrollable subsystems, an estimation on the solution is given by using the Lyapunov functional approach. Then, we show that the discretetime switched impulsive system is also stabilizable under appropriate switching signal when there exist uncontrollable subsystems.

This paper is organized as follows. In Section 2, some preliminaries are formulated. The main results of this paper 
are given in Section 3. Two examples are worked out in Section 4 to illustrate the main results. Section 5 concludes the paper.

\section{Preliminaries}

Consider the following planar discrete-time linear system:

$$
x(k+1)=G x(k)+H u(k), \quad k \geq 0,
$$

where $x \in R^{2}$ is the state, $u \in R^{m}$ is the controlled input, and $G$ and $H$ are matrices of appropriate dimensions.

Under the following linear feedback law:

$$
u(k)=K x(k)
$$

the solution of the system (1) takes the form

$$
x(k)=(G+H K)^{k} x(0),
$$

where $(G+H K)^{k}$ is called the transition matrix.

When the system (1) is controllable, we first establish an estimation on the transition matrix $(G+H K)^{k}$.

Lemma 1. Let $G \in R^{2 \times 2}$ and $H \in R^{2 \times m}$ be constant matrices such that the pair $(G, H)$ is controllable. Then, for any $0<\lambda<$ 1 , there exists a matrix $K \in R^{m \times 2}$ such that

$$
\left\|(G+H K)^{k}\right\| \leq N \lambda^{k-1}, \quad k \geq 0,
$$

where $N>0$ is a constant, which is independent of $\lambda$ and can be estimated precisely in terms of $G$ and $H$.

Proof. First, we consider the case of single input, that is, $m=$ 1. Noting that $(G, H)$ is controllable, we can choose a feedback matrix $K \in R^{1 \times 2}$ such that eigenvalues $\lambda_{i}$ of $G+H K$ satisfy

$$
0<\lambda_{i}<1, \quad \lambda_{i} \neq \lambda_{j}, \quad i \neq j, \quad i, j=1,2 .
$$

In particular, for any $0<\lambda<1$, we can choose

$$
\lambda_{i}=\frac{\lambda}{i}, \quad i=1,2
$$

Set

$$
F=\left[\begin{array}{ll}
G H & H
\end{array}\right] \times\left[\begin{array}{cc}
1 & 0 \\
a_{1} & 1
\end{array}\right]
$$

where $a_{i}, i=1,2$, are determined by

$$
\operatorname{det}(S I-G)=s^{2}+a_{1} s+a_{0} .
$$

Let

$$
G_{c}=F^{-1} G F, \quad H_{c}=F^{-1} H, \quad K_{c}=K F .
$$

We have that $\lambda_{i}, i=1,2$, are also eigenvalues of $\left(G_{c}+H_{c} K_{c}\right)$, and $\left(G_{c}, H_{c}\right)$ is in controller canonical form. Let

$$
P=\left[\begin{array}{cc}
1 & 1 \\
\lambda_{1} & \lambda_{2}
\end{array}\right] \text {. }
$$

It is not difficult to see that

$$
P^{-1}\left(G_{c}+H_{c} K_{c}\right) P=J=\operatorname{diag}\left\{\lambda_{1}, \lambda_{2}\right\},
$$

that is,

$$
P^{-1} F^{-1}(G+H K) F P=J .
$$

It implies that

$$
G+H K=F P J P^{-1} F^{-1}
$$

Consequently,

$$
\begin{aligned}
\left\|(G+H K)^{k}\right\| & =\left\|F P J^{k} P^{-1} F^{-1}\right\| \\
& \leq\|F\|\left\|F^{-1}\right\|\|P\|\left\|P^{-1}\right\|\left\|J^{k}\right\| .
\end{aligned}
$$

Let $P=\left[p_{i j}\right]$ and $P^{-1}=\left[e_{i j}\right]$. First, we have

$$
\|P\| \leq 2 \max _{1 \leq i, j \leq 2}\left\{\left|p_{i j}\right|\right\}=2 .
$$

Second, noting that $P^{-1}=\operatorname{adj} P / \operatorname{det} P$, we get from (6) that

$$
P^{-1}=\left[\begin{array}{cc}
-1 & \frac{2}{\lambda} \\
2 & \frac{-2}{\lambda}
\end{array}\right] \text {. }
$$

So,

$$
\left\|P^{-1}\right\| \leq \frac{4}{\lambda}
$$

Since

$$
\left\|J^{k}\right\| \leq \lambda_{1}^{k}=\lambda^{k}
$$

substituting (15), (17), and (18) into (14) yields that

$$
\left\|(G+H K)^{k}\right\| \leq\|F\|\left\|F^{-1}\right\| \times 2 \times \frac{4}{\lambda} \times \lambda^{k}=N \lambda^{k-1},
$$

where $N=8\|F\|\left\|F^{-1}\right\|$, which is independent of $\lambda$. Therefore, we have that Lemma 1 holds for the single input case.

For the multiple-input case, one sees that for any $c \in R^{m}$ such that $H c \neq 0$, there exists $K_{0} \in R^{m \times 2}$ such that $(G+$ $H K_{0}, H c$ ) is itself controllable. Hence, the conclusion of the single-input case that has been proved above is applicable to the controllable pair $\left(G+H K_{0}, H c\right)$. Therefore, for any $0<\lambda<1$, there exists $k_{1} \in R^{1 \times 2}$ such that

$$
\left\|\left(G+H K_{0}+H c k_{1}\right)^{k}\right\| \leq N \cdot \lambda^{k-1} .
$$

The proof of Lemma 1 is completed.

When $(G, H)$ is uncontrollable, for any given feedback $K$, there always exist a positive-definite symmetric matrix $P$ and an appropriate constant $\mu>0$ such that

$$
(G+H K)^{T} P(G+H K)-P<\mu P,
$$


which can be solved by using the GEVP solver in the LMI Toolbox of MATLAB [32].

Define the following Lyapunov function:

$$
V(x(k))=x^{T}(k) P x(k), \quad k \geq 0 .
$$

It is easy to see that

$$
\lambda_{\min }(P)\|x\|^{2} \leq V(x) \leq \lambda_{\max }(P)\|x\|^{2}
$$

where $\lambda_{\min }(P)$ and $\lambda_{\max }(P)$ denote the smallest and the largest eigenvalue of the positive definite symmetric matrix $P$.

Lemma 2. For system (1), if the pair $(G, H)$ is uncontrollable and (21) holds, then for any given feedback matrix $K$, there exists a constant $\mu>0$ such that

$$
\|x(k)\|<\left[\sqrt{\frac{(1+\mu) \lambda_{\max }(P)}{\lambda_{\min }(P)}}\right]^{k}\|x(0)\|, \quad k \geq 0 .
$$

Proof. Let the Lyapunov function be defined by (22). Along the solution of system (1), we have

$$
\begin{aligned}
\Delta V(x(k)) & =x^{T}(k+1) P x(k+1)-x^{T}(k) P x(k) \\
& =x^{T}(k)\left[(G+H K)^{T} P(G+H K)-P\right] x(k) .
\end{aligned}
$$

By (21) and (25), we obtain

$$
\begin{aligned}
\Delta V & (x(k))-\mu V(x(k)) \\
& =x^{T}(k)\left[(G+H K)^{T} P(G+H K)-P-\mu P\right] x(k)<0,
\end{aligned}
$$

which implies that

$$
V(x(k+1))<(1+\mu) V(x(k)) .
$$

By (23) and (27), we have

$$
\lambda_{\min }(P)\|x(k+1)\|^{2}<(1+\mu) \lambda_{\max }(P)\|x(k)\|^{2} .
$$

Thus,

$$
\|x(k+1)\|<\sqrt{\frac{(1+\mu) \lambda_{\max }(P)}{\lambda_{\min }(P)}}\|x(k)\| .
$$

By induction, we have

$$
\|x(k)\|<\left[\sqrt{\frac{(1+\mu) \lambda_{\max }(P)}{\lambda_{\min }(P)}}\right]^{k}\|x(0)\|, \quad k \geq 0 .
$$

This completes the proof of Lemma 2.

\section{Stabilization of Discrete-Time Planar Switched Impulsive Systems}

Now, we study the stabilization of the following discrete-time switched linear system:

$$
\begin{gathered}
x(k+1)=G_{\sigma(k)} x(k)+H_{\sigma(k)} u(k), \quad k \neq k_{j}, \\
x\left(k_{j}^{+}\right)=F\left(\sigma\left(k_{j}\right), \sigma\left(k_{j+1}\right)\right) x\left(k_{j}\right),
\end{gathered}
$$

where $x \in R^{2}$ is the state, $u \in R^{m}$ is the input, and $\sigma(k) \in \Lambda=$ $\{1,2, \ldots, N\}$ is a switching signal for some positive integer $N>1$, which is a piecewise constant function. When $\sigma(k)=$ $i, i \in \Lambda$, system (31) switches to the $i$ th subsystem. Moreover, $F(\cdot, \cdot)$ is a constant matrix, representing the impulse effect on the system at the switching time. Moreover, $0=k_{0}<$ $k_{1}<k_{2}<\cdots$ denote the discontinuous points (or switching points) of $\sigma(k)$, and denote $\sigma\left(k_{j-1}\right)=p_{j},(j=1,2, \ldots) . G_{i}$ and $H_{i}, i \in \Lambda$, are system matrices of appropriate dimensions.

Throughout this paper, we assume that

(H1) $\max _{1 \leq i, j \leq N}\|F(i, j)\| \leq \delta$, where $\delta>0$ is a positive constant.

Under the linear feedback law $u(k)=K_{\sigma(k)} x(k)$ for $k \neq k_{j}$, $j=1,2 \ldots$, system (31) reduces to the following closed-loop system:

$$
x(k+1)=\left[G_{\sigma(k)}+H_{\sigma(k)} K_{\sigma(k)}\right] x(k), \quad k \neq k_{j} .
$$

Denote the frequency of the switching signal by

$$
f=\lim \sup _{k \rightarrow \infty} \frac{N_{\sigma}(0, k)}{k},
$$

where $N_{\sigma}(0, k)$ is the number of activated subsystems on $[0, k]$. If $\left(G_{i}, H_{i}\right)$ is controllable for $i \in \Lambda$, we have the following result.

Theorem 3. Assume that (H1) and (H2) hold and $\left(G_{i}, H_{i}\right)$ is controllable for $i \in \Lambda$. Then, there exist a set of feedback matrices $\left\{K_{i}\right\}_{i \in \Lambda}$ such that the closed-loop system (32) is asymptotically stable for any switching signal $\sigma$ with a frequency $f<1$.

Proof. For any $k>0$, assume that $k \in\left(k_{i}, k_{i+1}\right]$ for some positive integer $i$. Note that $f<1$. By the definition of $f$, we can choose a constant $0<\alpha<1$ such that $i+1 \leq k \alpha$ for sufficiently large $k$. Without loss of generality, we assume that $i+1 \leq k \alpha$ for $k \geq 0$. Set $\beta=1-\alpha$. It is easy to see that $\beta>0$. Let $c=(\delta N)^{\alpha / \beta}$ and $\lambda$ be sufficiently small such that $\lambda c<1$. For such a choice of $\lambda$, by Lemma 1 , there exist a set of feedback matrices $\left\{K_{i}\right\}_{i \in \Lambda}$ such that for any $k>\widetilde{k} \geq 0$,

$$
\left\|\left(G_{i}+H_{i} K_{i}\right)^{k-\tilde{k}}\right\| \leq N \lambda^{k-\tilde{k}-1}
$$


where $N=\max _{i \in \Lambda} N_{i}$. For any $l \in\left[k_{j-1}, k_{j}\right),(1 \leq j \leq i+1)$, we have

$$
\begin{aligned}
\sigma(l) & =\sigma\left(k_{j-1}\right)=p_{j}, \\
x(l+1) & =\left(G_{p_{j}}+H_{p_{j}} K_{p_{j}}\right) x(l), \\
x\left(k_{j}^{+}\right) & =F\left(p_{j}, p_{j+1}\right) x\left(k_{j}\right), \\
x\left(k_{j}\right) & =\left(G_{p_{j}}+H_{p_{j}} K_{p_{j}}\right)^{k_{j}-k_{j-1}} x\left(k_{j-1}^{+}\right) .
\end{aligned}
$$

Since $k_{i} \leq k<k_{i+1}$, that is, $k \in\left[k_{i}, k_{i+1}\right)$, we obtain

$$
\begin{aligned}
x(k)= & \left(G_{p_{i+1}}+H_{p_{i+1}} K_{p_{i+1}}\right)^{k-k_{i}} x\left(k_{i}^{+}\right) \\
= & \left(G_{p_{i+1}}+H_{p_{i+1}} K_{p_{i+1}}\right)^{k-k_{i}} F\left(p_{i}, p_{i+1}\right) \\
& \times\left(G_{p_{i}}+H_{p_{i}} K_{p_{i}}\right)^{k_{i}-k_{i-1}} x\left(k_{i-1}^{+}\right) \\
= & \left(G_{p_{i+1}}+H_{p_{i+1}} K_{p_{i+1}}\right)^{k-k_{i}} F\left(p_{i}, p_{i+1}\right) \\
& \times\left(G_{p_{i}}+H_{p_{i}} K_{p_{i}}\right)^{k_{i}-k_{i-1}} \\
& \times F\left(p_{i-1}, p_{i}\right)\left(G_{p_{i-1}}+H_{p_{i-1}} K_{p_{i-1}}\right)^{k_{i-1}-k_{i-2}} x\left(k_{i-2}^{+}\right) \\
= & \left(G_{p_{i+1}}+H_{p_{i+1}} K_{p_{i+1}}\right)^{k-k_{i}} \prod_{j=1}^{i} F\left(p_{j}, p_{j+1}\right) \\
& \times\left(G_{p_{j}}+H_{p_{j}} K_{p_{j}}\right)^{k_{j}-k_{j-1}} x(0) .
\end{aligned}
$$

By the analysis and (H1), we obtain

$$
\begin{aligned}
\|x(k)\| \leq & \left\|\left(G_{p_{i+1}}+H_{p_{i+1}} K_{p_{i+1}}\right)^{k-k_{i}}\right\| \times \prod_{j=1}^{i}\left\|F\left(p_{j}, p_{j+1}\right)\right\| \\
& \times\left\|\left(G_{p_{j}}+H_{p_{j}} K_{p_{j}}\right)^{k_{j}-k_{j-1}}\right\| \times\|x(0)\| \\
\leq & \delta^{i} N^{i+1} \lambda^{k-i-1}\|x(0)\| \\
\leq & \delta^{i+1} N^{i+1} \lambda^{-(i+1)} \lambda^{k}\|x(0)\| \\
\leq & \left(\delta N \lambda^{-1}\right)^{k \alpha} \lambda^{k}\|x(0)\| \\
= & (\delta N)^{\alpha k} \lambda^{\beta k}\|x(0)\| \\
= & (\lambda c)^{\beta k}\|x(0)\| .
\end{aligned}
$$

Noting that $\lambda c<1$ and $\beta>0$, we have that system (31) is stabilizable under arbitrary switching signal with a frequency $f<1$. This completes the proof of Theorem 3 .

Next, we consider the case when there exist both controllable subsystems and uncontrollable subsystems for system (31). For the sake of convenience, we suppose that
(H2) $\left(G_{1}, H_{1}\right),\left(G_{2}, H_{2}\right), \ldots,\left(G_{p}, H_{p}\right)$ are uncontrollable subsystems and $\left(G_{p+1}, H_{p+1}\right),\left(G_{p+2}, H_{p+2}\right), \ldots$, $\left(G_{I}, H_{I}\right)$ are controllable subsystems, where $1 \leq$ $p<I$.

Denote the switching frequency of those controllable subsystems by

$$
\widetilde{f}=\lim \sup _{k \rightarrow \infty} \frac{\widetilde{N}_{\sigma}(0, k)}{k},
$$

where $\widetilde{N}_{\sigma}(0, k)$ is the number of activated controllable subsystems on $[0, k]$. Denote the total activation time for those controllable subsystems on $[0, k]$ by $T(0, k)$. In this paper, we assume that there exists a constant $\gamma>0$ such that

$$
T(0, k) \geq \gamma k, \quad k \geq 0 .
$$

Similar to the above analysis, for any given feedback matrices $K_{i}(1 \leq i \leq p)$, there exist positive definite symmetric matrices $P_{i}$ and positive constants $\mu_{i}$ such that

$$
\left(G_{i}+H_{i} K_{i}\right)^{T} P_{i}\left(G_{i}+H_{i} K_{i}\right)-P_{i}<\mu_{i} P_{i}, \quad 1 \leq i \leq p .
$$

Set

$$
\tilde{\lambda}=\max _{1 \leq i \leq p}\left\{\sqrt{\frac{\left(1+\mu_{i}\right) \lambda_{\max }\left(P_{i}\right)}{\lambda_{\min }\left(P_{i}\right)}}\right\}>1 .
$$

Theorem 4. Assume that (H2), (39), and (40) hold. Then, there exist a set of feedback matrices $\left\{K_{i}\right\}_{i \in \Lambda}$ such that the closed-loop system (32) is asymptotically stable for any switching signal $\sigma$ with a frequency $\tilde{f}<\gamma$.

Proof. For any $k>0$, assume that $k \in\left[k_{i}, k_{i+1}\right)$ for some positive integer $i$. Denote the number of activated controllable subsystems in $[0, k]$ by $i_{1}$. Since $\tilde{f}<\gamma$. By the definition of $\tilde{f}$, there exists a constant $0<\tilde{\alpha}<\gamma$ such that $i_{1} \leq k \widetilde{\alpha}$ for $k \geq 0$ without loss of generality. Set $\widetilde{\beta}=\gamma-\widetilde{\alpha}$. It is easy to see that $\widetilde{\beta}>0$ since $0<\widetilde{\alpha}<\gamma$. Let the constant $\widetilde{c}$ satisfies

$$
\delta N^{\tilde{\alpha}} \tilde{\lambda}=\widetilde{c}^{\widetilde{\beta}},
$$

where $\tilde{\lambda}$ is determined by (41) under any given feedback matrices $\left\{K_{i}\right\}_{1 \leq i \leq p}$.

For any $k \geq 0$, assume that $\sigma\left(k_{j-1}\right)=p_{j}, 1 \leq j \leq i+1$ and $k_{i} \leq k<k_{i+1}$. By (35), we have

$$
\begin{aligned}
x(k) & =\left(G_{p_{i+1}}+H_{p_{i+1}} K_{p_{i+1}}\right)^{k-k_{i}} x\left(k_{i}^{+}\right) \\
& =\left(G_{p_{i+1}}+H_{p_{i+1}} K_{p_{i+1}}\right)^{k-k_{i}} F\left(p_{i}, p_{i+1}\right) x\left(k_{i}\right) .
\end{aligned}
$$

If $p_{j} \in\{p+1, p+2, \ldots, N\}$, by (34) and (H1), we have

$$
\begin{aligned}
\|x(k)\| \leq & \left\|\left(G_{p_{i+1}}+H_{p_{i+1}} K_{p_{i+1}}\right)^{k-k_{i}}\right\| \\
& \cdot\left\|F\left(p_{i}, p_{i+1}\right)\right\| \cdot\left\|x\left(k_{i}\right)\right\| \\
\leq & \delta N \lambda^{k-k_{i}-1}\left\|x\left(k_{i}\right)\right\| .
\end{aligned}
$$


If $p_{j} \in\{1,2, \ldots, p\}$, by Lemma $2,(41)$, and (H1), we have

$$
\begin{aligned}
\|x(k)\| & \leq\left(\sqrt{\frac{(1+\mu) \lambda_{\max }(P)}{\lambda_{\min }(P)}}\right)^{k-k_{i}} \delta\left\|x\left(k_{i}\right)\right\| \\
& \leq \delta \widetilde{\lambda}^{k-k_{i}}\left\|x\left(k_{i}\right)\right\| .
\end{aligned}
$$

Based on the same analysis, there exists a feedback matrix $K_{p_{m-1}}$ for each $m \in\{1,2, \ldots, j\}$ such that

$$
\left\|x\left(k_{m}\right)\right\| \leq\left\{\begin{array}{c}
\delta N \lambda^{k_{m}-k_{m-1}}\left\|x\left(k_{m-1}\right)\right\|, \\
\sigma\left(k_{m-1}\right) \in\{p+1, p+2, \ldots, N\}, \\
\delta \widetilde{\lambda}^{k_{m}-k_{m-1}\left\|x\left(k_{m-1}\right)\right\|,} \\
\sigma\left(k_{m-1}\right) \in\{1,2, \ldots, p\} .
\end{array}\right.
$$

We now choose $\lambda$ sufficiently small such that $\lambda \widetilde{c}<1$. Under the feedback law $u(k)=K_{i_{m}} x(t)$ for $m=0,1, \ldots, j$, we get from (39) and (44)-(46) by induction that

$$
\begin{aligned}
\|x(k)\| & \leq \delta^{k}\left[N \lambda^{-1}\right]^{i_{1}} \lambda^{T(0, k)} \widetilde{\lambda}^{k}\|x(0)\| \\
& \leq \delta^{k}\left[N \lambda^{-1}\right]^{\widetilde{\alpha} k} \lambda^{\gamma k} \widetilde{\lambda}^{k}\|x(0)\| \\
& =\left(\delta N^{\tilde{\alpha}} \widetilde{\lambda}\right)^{k} \lambda^{\tilde{\beta} k}\|x(0)\| \\
& =(\widetilde{c} \lambda)^{\tilde{\beta} k}\|x(0)\| .
\end{aligned}
$$

Noting that $\tilde{c} \lambda<1$ and $\widetilde{\beta}>0$, we have that system (32) is stabilizable under arbitrary switching signal with a frequency $\widetilde{f}<\gamma$. This completes the proof of Theorem 4 .

\section{Examples}

In order to illustrate the theoretical result, we consider two examples.

Example 1. Consider the switched systems (31), with $\sigma(k)=$ $1,2,3$, and

$$
\begin{array}{ll}
G_{1}=\left[\begin{array}{ll}
3 & 1 \\
0 & 5
\end{array}\right], & H_{1}=\left[\begin{array}{l}
1 \\
4
\end{array}\right], \\
G_{2}=\left[\begin{array}{cc}
7 & 4 \\
10 & -1
\end{array}\right], & H_{2}=\left[\begin{array}{l}
3 \\
1
\end{array}\right], \\
G_{3}=\left[\begin{array}{ll}
8 & 3 \\
5 & 1
\end{array}\right], & H_{3}=\left[\begin{array}{l}
5 \\
1
\end{array}\right] .
\end{array}
$$

It is not difficult to verify that $\left(G_{i}, H_{i}\right)$ is controllable for $i=$ $1,2,3$.

By Lemma 1, we get $N=\max _{i=1,2,3} N_{i}=161$. We choose $\lambda=1 / 2$, then the closed-loop poles of $(32)$ are $1 / 2$ and $1 / 4$. We have the feedback matrices

$$
\begin{aligned}
& K_{1}=\left[\begin{array}{ll}
3.4375 & 0.9531
\end{array}\right], \\
& K_{2}=\left[\begin{array}{ll}
1.1875 & 1.6875
\end{array}\right], \\
& K_{3}=\left[\begin{array}{ll}
1.5302 & 0.5991
\end{array}\right] .
\end{aligned}
$$

Let $\delta=2$ and the switching frequency $f<1$. Based on the proof of Theorem 3, we can choose that $\alpha=1 / 20$, then

$$
\begin{aligned}
& \beta=1-\alpha=\frac{19}{20}>0, \\
& c=(\delta N)^{\alpha / \beta}=1.3552 .
\end{aligned}
$$

We can get $\lambda c=0.6776<1$. By Theorem 3 , the system (32) is asymptotically stable with $f<1$.

Example 2. Consider the switched systems (31), with $\sigma(k)=$ $1,2,3$, and

$$
\begin{array}{ll}
G_{1}=\left[\begin{array}{cc}
0 & 1 \\
-1 & 0
\end{array}\right], & H_{1}=\left[\begin{array}{l}
1 \\
0
\end{array}\right], \\
G_{2}=\left[\begin{array}{cc}
1 & -4 \\
2 & 0
\end{array}\right], & H_{2}=\left[\begin{array}{l}
2 \\
1
\end{array}\right], \\
G_{3}=\left[\begin{array}{ll}
1 & 0 \\
0 & 1
\end{array}\right], & H_{3}=\left[\begin{array}{l}
1 \\
0
\end{array}\right] .
\end{array}
$$

It is not difficult to verify that $\left(G_{i}, H_{i}\right)$ is controllable for $i=$ 1,2 , and $\left(G_{3}, H_{3}\right)$ is uncontrollable.

By Lemma 1, we get $N=\max _{i=1,2} N_{i}=21$. Choose $\lambda=$ $1 / 10$, we have the feedback matrices

$$
\begin{aligned}
& K_{1}=\left[\begin{array}{ll}
-0.1500 & 0.9950
\end{array}\right], \\
& K_{2}=\left[\begin{array}{ll}
1.0545 & -1.2590
\end{array}\right] .
\end{aligned}
$$

Let $\delta=3$ and $\gamma=3$. The switching frequency $\tilde{f}<3, \widetilde{\alpha}=1 / 10$. For given $K_{3}=\left[\begin{array}{ll}2 & 1\end{array}\right]$. Based on the (40), we can choose $\mu_{3}=$ 12 and $P_{3}=\left[\begin{array}{ll}3 & 1 \\ 1 & 1\end{array}\right]$, then

$$
\widetilde{\lambda}=25.0889 \text {. }
$$

By Theorem 4, we have

$$
\begin{aligned}
\widetilde{\beta} & =\frac{29}{10}>0, \\
\widetilde{c} & =4.92,
\end{aligned}
$$

then

$$
\lambda \widetilde{c}=0.492<1
$$

By Theorem 4, the system (32) is asymptotically stable with $\tilde{f}<3$.

\section{Conclusion}

In this paper, the stabilization problem of discrete-time planar switched linear systems with impulse is investigated. When all the subsystems are controllable, we first establish an estimation on the transition matrix for each controllable subsystem, which is a discrete analogue of the corresponding result in [17]. By using such an estimation, we prove that the discrete-time switched impulsive system is stabilizable under arbitrary switching signal with a given switching frequency. 
When there exists at least one uncontrollable subsystem, by using a Lyapunov functional approach, we show that the stabilizability of the switched impulsive system can be retained for the appropriate switching frequency of those controllable subsystems.

\section{Acknowledgments}

The authors thank the reviewers for their helpful and valuable comments on this paper. This work was supported by the Natural Science Foundation of Shandong Province under Grant nos. JQ201119 and ZR2010AL002 and the National Natural Science Foundation of China under Grant no. 61174217.

\section{References}

[1] D. Liberzon, Switching in Systems and Control, Systems \& Control: Foundations \& Applications, Birkhäuser, Boston, Mass, USA, 2003.

[2] Z. Sun and S. S. Ge, Switched Linear Systems: Control and Design, Springer, New York, NY, USA, 2005.

[3] Z. Sun and S. S. Ge, Stability Theory of Switched Dynamical Systems, Springer, London, UK, 2011.

[4] D. Liberzon and A. S. Morse, "Basic problems in stability and design of switched systems," IEEE Control Systems Magazine, vol. 19, no. 5, pp. 59-70, 1999.

[5] H. Lin and P. J. Antsaklis, "Stability and stabilizability of switched linear systems: a survey of recent results," IEEE Transactions on Automatic Control, vol. 54, no. 2, pp. 308-322, 2009.

[6] A. Gollu and P. Varaiya, "Hybrid dynamical systems," in Proceedings of the 28th Conference on Decision and Control, pp. 2708-2712, Tampa, Fla, USA, 1989.

[7] M. Dogruel, S. Drakunov, and U. Ozguner, "Sliding mode control in discrete state systems," in Proceedings of the 32nd IEEE Conference on Decision and Control, pp. 1194-1199, 1993.

[8] I. A. Hiskens, "Analysis tools for power systems-contending with nonlinearities," Proceedings of the IEEE, vol. 83, no. 11, pp. 1573-1587, 1995.

[9] C. Tomlin, G. J. Pappas, and S. Sastry, "Conflict resolution for air traffic management: a study in multiagent hybrid systems," IEEE Transactions on Automatic Control, vol. 43, no. 4, pp. 509$521,1998$.

[10] J. Lygeros, D. N. Godbole, and S. Sastry, "Verified hybrid controllers for automated vehicles," IEEE Transactions on Automatic Control, vol. 43, no. 4, pp. 522-539, 1998.

[11] D. Liberzon, J. P. Hespanha, and A. S. Morse, "Stability of switched systems: a Lie-algebraic condition," Systems \& Control Letters, vol. 37, no. 3, pp. 117-122, 1999.

[12] A. A. Agrachev and D. Liberzon, "Lie-algebraic stability criteria for switched systems," SIAM Journal on Control and Optimization, vol. 40, no. 1, pp. 253-269, 2001.

[13] D. Cheng, L. Guo, and J. Huang, "On quadratic Lyapunov functions," IEEE Transactions on Automatic Control, vol. 48, no. 5, pp. 885-890, 2003.

[14] R. Shorten, K. S. Narendra, and O. Mason, "A result on common quadratic Lyapunov functions," IEEE Transactions on Automatic Control, vol. 48, no. 1, pp. 110-113, 2003.

[15] X. Liu, "Stabilization of switched linear systems with modedependent time-varying delays," Applied Mathematics and Computation, vol. 216, no. 9, pp. 2581-2586, 2010.
[16] D. Cheng, L. Guo, Y. Lin, and Y. Wang, "Stabilization of switched linear systems," IEEE Transactions on Automatic Control, vol. 50, no. 5, pp. 661-666, 2005.

[17] Y. G. Sun, L. Wang, G. Xie, and M. Yu, "Improved overshoot estimation in pole placements and its application in observerbased stabilization for switched systems," IEEE Transactions on Automatic Control, vol. 51, no. 12, pp. 1962-1966, 2006.

[18] Y. Sun, "Stabilization of switched systems with nonlinear impulse effects and disturbances," IEEE Transactions on Automatic Control, vol. 56, no. 11, pp. 2739-2743, 2011.

[19] B. Du and X. Zhang, "Delay-dependent stability analysis and synthesis for uncertain impulsive switched system with mixed delays," Discrete Dynamics in Nature and Society, vol. 2011, Article ID 381571, 9 pages, 2011.

[20] M. de la Sen and A. Ibeas, "Stability results of a class of hybrid systems under switched continuous-time and discretetime control," Discrete Dynamics in Nature and Society, vol. 2009, Article ID 315713, 28 pages, 2009.

[21] M. Rajchakit and G. Rajchakit, "Mean square exponential stability of stochastic switched system with interval timevarying delays," Abstract and Applied Analysis, vol. 2012, Article ID 623014, 12 pages, 2012.

[22] Y. G. Sun, L. Wang, and G. Xie, "Necessary and sufficient conditions for stabilization of discrete-time planar switched systems," Nonlinear Analysis: Theory, Methods \& Applications, vol. 65, no. 5, pp. 1039-1049, 2006.

[23] Y. G. Sun, L. Wang, and G. Xie, "Delay-dependent robust stability and stabilization for discrete-time switched systems with mode-dependent time-varying delays," Applied Mathematics and Computation, vol. 180, no. 2, pp. 428-435, 2006.

[24] Y. G. Sun, L. Wang, and G. Xie, "Delay-dependent robust stability and $H_{\infty}$ control for uncertain discrete-time switched systems with mode-dependent time delays," Applied Mathematics and Computation, vol. 187, no. 2, pp. 1228-1237, 2007.

[25] Y. Song, J. Fan, M. Fei, and T. Yang, "Robust $H_{\infty}$ control of discrete switched system with time delay," Applied Mathematics and Computation, vol. 205, no. 1, pp. 159-169, 2008.

[26] S. Ibrir, "Stability and robust stabilization of discrete-time switched systems with time-delays: LMI approach," Applied Mathematics and Computation, vol. 206, no. 2, pp. 570-578, 2008.

[27] S. Ma, C. Zhang, and Z. Wu, "Delay-dependent stability of $H_{\infty}$ control for uncertain discrete switched singular systems with time-delay," Applied Mathematics and Computation, vol. 206, no. 1, pp. 413-424, 2008.

[28] Y. G. Sun, L. Wang, and G. Xie, "Necessary and sufficient conditions for stabilization of discrete-time planar switched systems," Nonlinear Analysis: Theory, Methods \& Applications, vol. 65, no. 5, pp. 1039-1049, 2006.

[29] G. Zong, L. Hou, and Y. Wu, "Exponential $l_{2}-l_{\infty}$ filtering for discrete-time switched systems under a new framework," International Journal of Adaptive Control and Signal Processing, vol. 26, no. 2, pp. 124-137, 2012.

[30] G. Zong, L. Hou, and Y. Wu, "Robust $l_{2}-l_{\infty}$ guaranteed cost filtering for uncertain discrete-time switched system with mode-dependent time-varying delays," Circuits, Systems, and Signal Processing, vol. 30, no. 1, pp. 17-33, 2011.

[31] G. Zong, S. Xu, and Y. Wu, "Robust $H_{\infty}$ stabilization for uncertain switched impulsive control systems with state delay: an LMI approach," Nonlinear Analysis: Hybrid Systems, vol. 2, no. 4, pp. 1287-1300, 2008. 
[32] G. Gahinet, A. Nemirovski, A. J. Laub, and M. Chilali, LMI Control Toolbox for Use with Matlab, The MathWorks Inc., Natick, Mass, USA, 1995. 


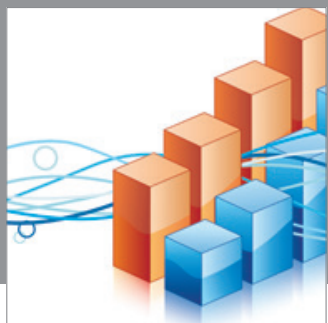

Advances in

Operations Research

mansans

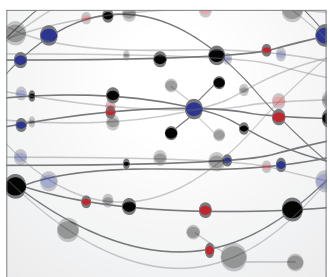

The Scientific World Journal
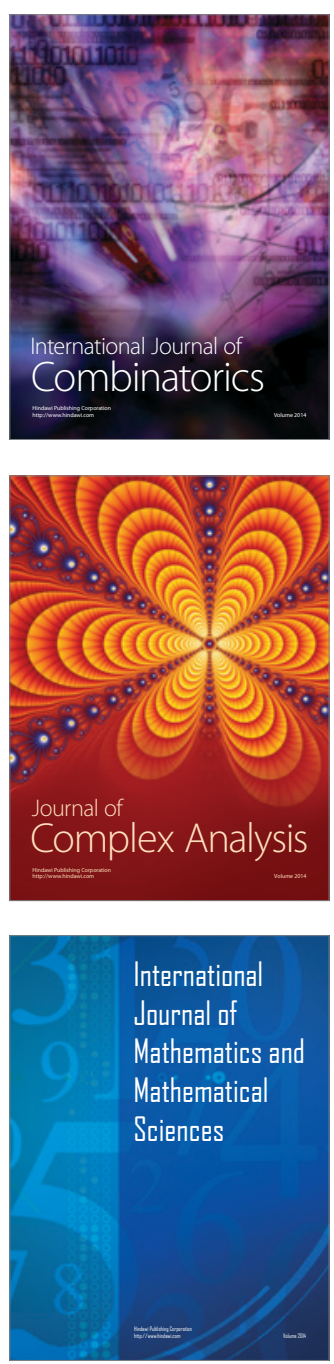
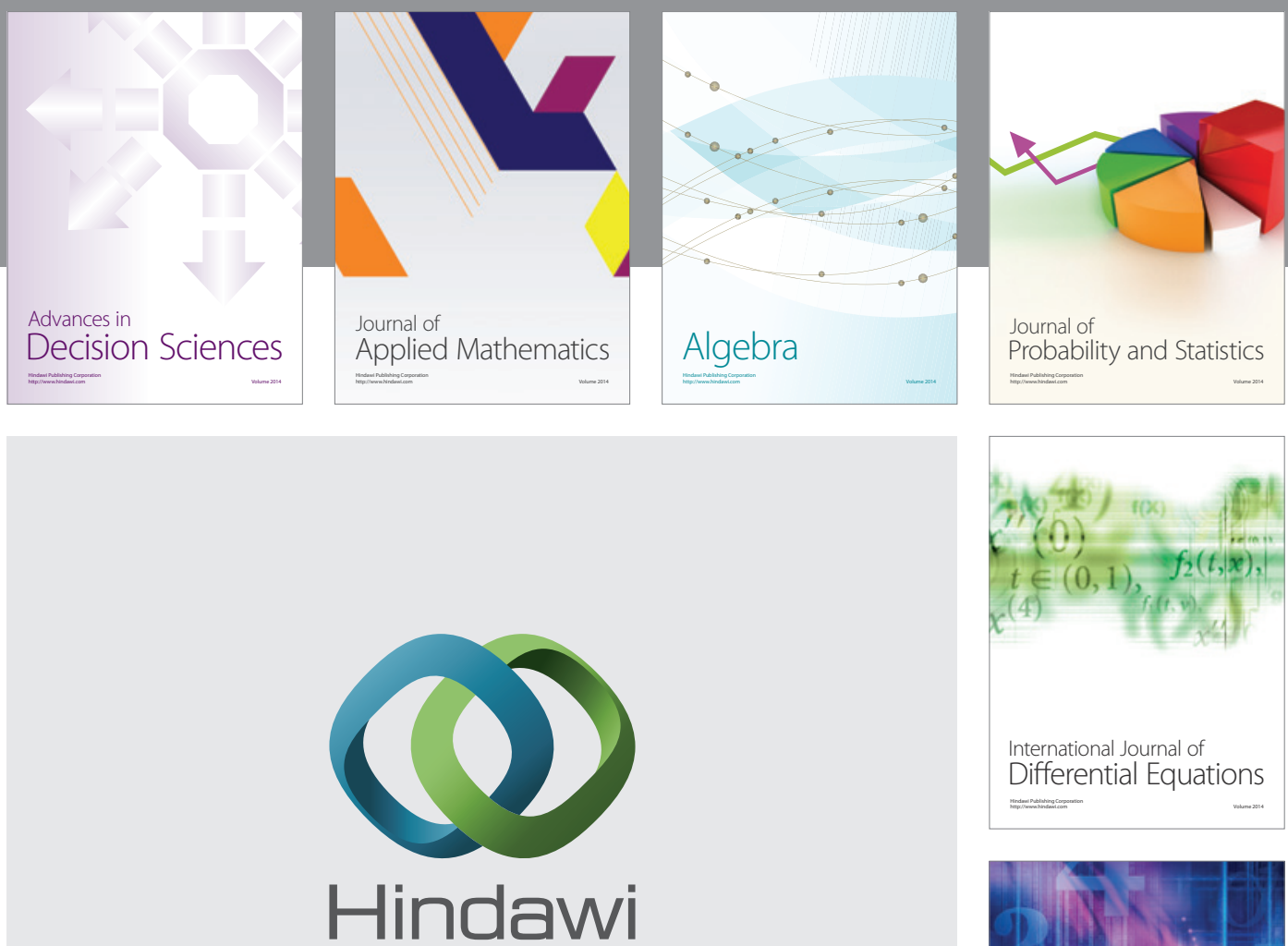

Submit your manuscripts at http://www.hindawi.com
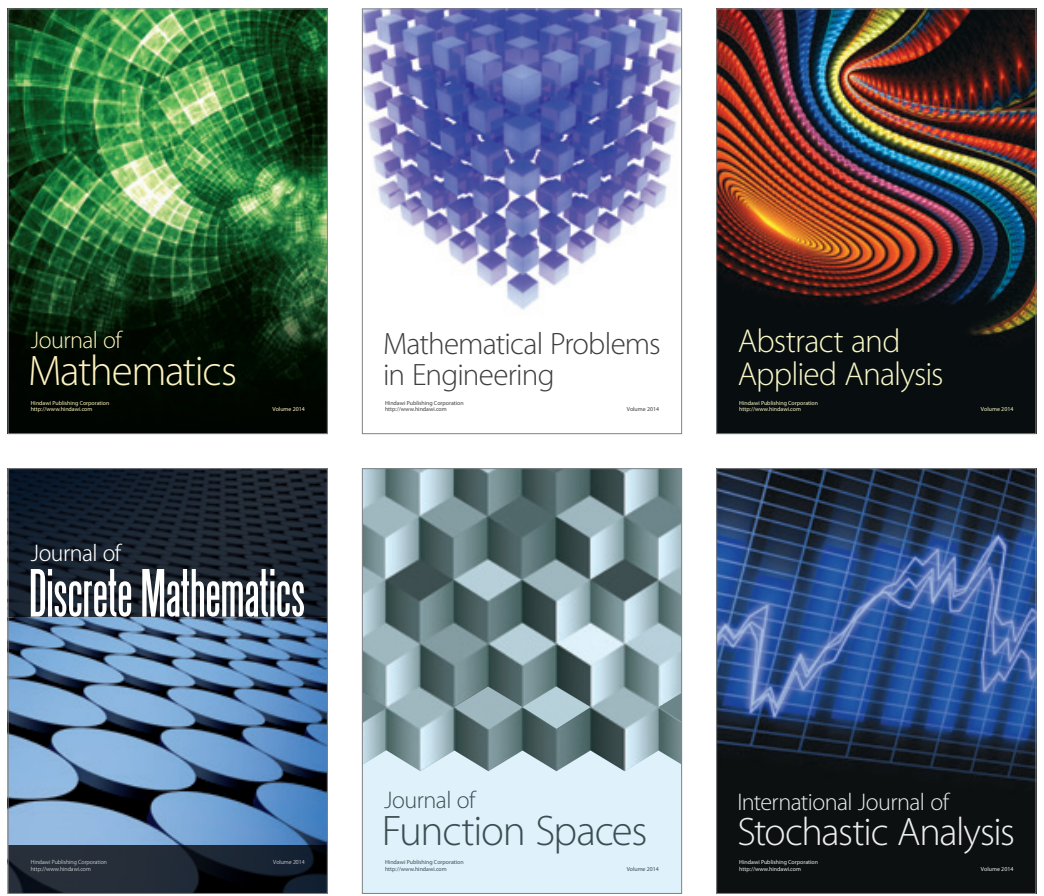

Journal of

Function Spaces

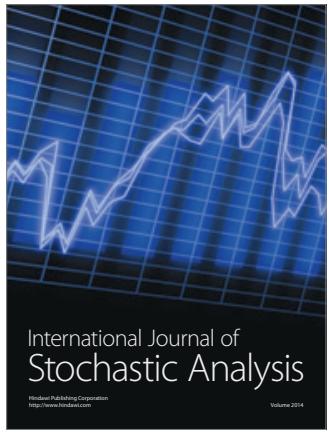

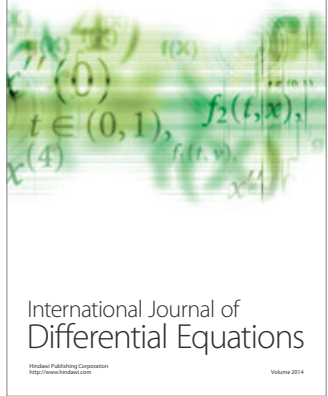
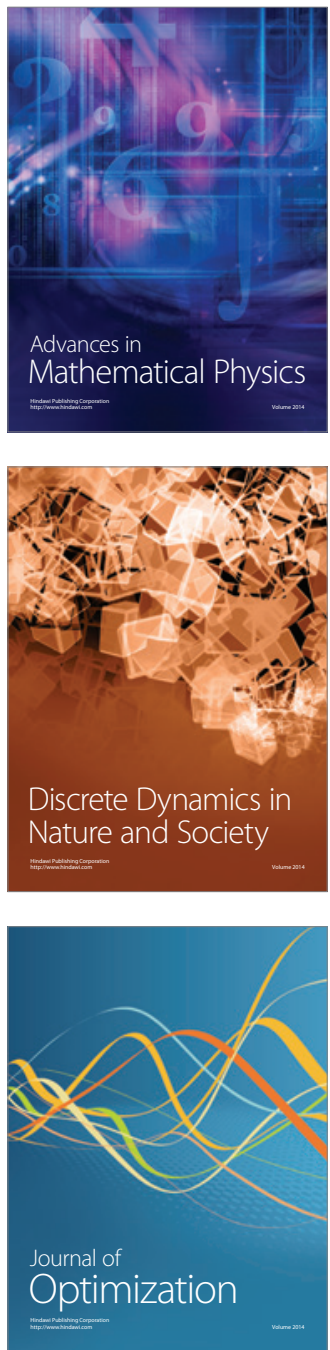